\title{
An evaluation of the sonoporation potential of low-boiling point phase-change ultrasound contrast agents in vitro
}

\author{
Samantha M. Fix ${ }^{1}$, Anthony Novell ${ }^{2}$, Yeoheung Yun ${ }^{3}$, Paul A. Dayton ${ }^{1,2}$ and Christopher B. Arena $a^{2,4^{*}}$
}

\begin{abstract}
Background: Phase-change ultrasound contrast agents (PCCAs) offer a solution to the inherent limitations associated with using microbubbles for sonoporation; they are characterized by prolonged circulation lifetimes, and their nanometer-scale sizes may allow for passive accumulation in solid tumors. As a first step towards the goal of extravascular cell permeabilization, we aim to characterize the sonoporation potential of a low-boiling point formulation of PCCAs in vitro.

Methods: Parameters to induce acoustic droplet vaporization and subsequent microbubble cavitation were optimized in vitro using high-speed optical microscopy. Sonoporation of pancreatic cancer cells in suspension was then characterized at a range of pressures (125-600 $\mathrm{kPa}$ ) and pulse lengths (5-50 cycles) using propidium iodide as an indicator molecule.

Results: We achieved sonoporation efficiencies ranging from $8 \pm 1 \%$ to $36 \pm 4 \%$ (percent of viable cells), as evidenced by flow cytometry. Increasing sonoporation efficiency trended with increasing pulse length and peak negative pressure.

Conclusions: We conclude that PCCAs can be used to induce the sonoporation of cells in vitro, and our results warrant further investigation into the use of PCCAs as extravascular sonoporation agents in vivo.
\end{abstract}

Keywords: Sonoporation, Ultrasound, Drug delivery, Acoustic droplet vaporization, Nanodroplet

\section{Background}

Sonoporation refers to the process by which ultrasoundstimulated microbubbles are used to permeabilize cell membranes and enhance the intracellular accumulation of drugs, genes, or indicator dyes [1, 2]. This holds potential as a physical targeting method to drive drug delivery non-invasively and with high spatial specificity. However, inherent limitations associated with microbubble contrast agents used for previous in vitro investigations [3-7] must be overcome to enable in vivo

\footnotetext{
* Correspondence: carena3@elon.edu

${ }^{2}$ Joint Department of Biomedical Engineering, University of North Carolina

Chapel Hill and North Carolina State University, Chapel Hill, NC, USA

${ }^{4}$ Laboratory for Therapeutic Directed Energy, Department of Physics, Elon

University, Elon, NC, USA

Full list of author information is available at the end of the article
}

translation and subsequent widespread utility of this technique.

First, microbubbles are relatively large $(1-10 \mu \mathrm{m})$ and therefore cannot escape the vasculature following intravenous administration [8]. This can be useful for some applications including drug or gene delivery to vascular endothelial cells [9] and ultrasound-mediated disruption of endothelial tight junctions to open the blood-brain barrier $[10,11]$. However, extravascular sonoporation for the purpose of improved drug or gene delivery within a target tissue is not feasible. Second, microbubbles have limited persistence in circulation $(<10 \mathrm{~min})$ [8]. This necessitates continuous infusion or repeat bolus injections in situations where repeat or long duration treatment is required.

Phase-change ultrasound contrast agents (PCCAs) are nanometer scale, liquid-filled droplets that can be 
vaporized into microbubbles when subjected to ultrasound of sufficient amplitude through a process termed acoustic droplet vaporization (ADV). These agents are characterized by longer circulation half-lives than similarly formulated microbubbles [12, 13], and their nanometer-scale size distributions may allow for passive accumulation in leaky tumors via the enhanced permeability and retention (EPR) effect [13, 14]. Furthermore, since PCCAs are nearly invisible to ultrasound in their liquid state, high concentrations can be used without the shielding effect characteristic of high microbubble concentrations. PCCA-derived microbubbles destroyed in one acoustic pulse may be replenished through subsequent vaporization events, thereby allowing for the sustained generation of cavitation energy and enhanced sonoporation [15]. PCCAs therefore offer a solution to the major limitations previously given for microbubblemediated sonoporation and hold the potential for extravascular sonoporation in vivo.

PCCA formulations are commonly filled with perfluorocarbons with boiling points near body temperature, such as dodecafluoropentane (DDFP, bp $=29{ }^{\circ} \mathrm{C}$ ), and a few laboratories have demonstrated the sonoporation potential of such agents in vitro [15-18]. While these initial studies show promise, the high negative pressures required to vaporize nanoscale DDFP-filled PCCAs (3-6 MPa [14, 15]) may cause unwanted bioeffects such as heating or cell lysis in an in vivo setting. Our laboratory has developed a class of low-boiling point PCCAs filled with octofluoropropane (OFP, $\mathrm{bp}=-36.7^{\circ} \mathrm{C}$ ), which are characterized by far lower pressure requirements for vaporization when compared to DDFP-filled PCCAs $(\sim 20 \times$ lower). Therefore, we hypothesize that our formulation will offer greater control over the bioeffects caused by $\mathrm{ADV}$ and subsequent microbubble cavitation. The primary objective of this study is to characterize the sonoporation potential of these low-boiling point PCCAs in vitro.

The precise mechanisms involved in PCCA-mediated sonoporation remain unknown, and likely depend on a number of factors including the contrast agent formulation, specific acoustic parameters (frequency, peak negative pressure [3], duty cycle, etc.), and non-acoustic parameters (microbubble size and bubble-to-cell distance [19], cell culture conditions, size of sonoporation indicator [3], etc.). It is conceivable that PCCA-induced sonoporation is driven by the same mechanisms that mediate microbubble sonoporation, with membrane permeabilization being a product of microbubble cavitation following ADV. However, the rapid expansion of an individual droplet as it phase-converts into a microbubble may itself influence cell permeability. A secondary objective of this study is to determine if the vaporization event of low-boiling point PCCAs contributes to sonoporation and/or effects cell viability.

\section{Methods}

\section{Fabrication and characterization of phase-change ultrasound contrast agents}

Low-boiling point PCCAs containing liquid octafluoropropane (OFP, boiling point $-36.7{ }^{\circ} \mathrm{C}$ ) were generated as described elsewhere [20]. First, lipidshelled, OFP-filled microbubbles were prepared. Briefly, 90 mol\% 1,2-distearoyl-sn-glycero-3-phosphocholine (DSPC) and $10 \mathrm{~mol} \%$ 1,2-distearoyl-sn-glycero-3-phosphoethanolamine- $N$-methoxy(polyethylene-glycol)-2000 (DSPEPEG2000) (Avanti Polar Lipids, Alabaster, AL, USA) were combined and dissolved in a phosphate-buffered saline (PBS)-based solution containing 15\% propylene glycol $(v / v)$ and $5 \%$ glycerol $(v / v)$ for a final lipid concentration of $1.0 \mathrm{mg} / \mathrm{mL}$. This lipid solution $(1.5 \mathrm{~mL})$ was aliquoted into $3.0 \mathrm{~mL}$ glass vials and the headspace air was exchanged with OFP gas (FluoroMed, Round Rock, TX, USA). Finally, microbubbles were generated by vigorous shaking of the lipid vials using a VialMix (Bristol-Myers-Squibb, New York, NY, USA).

The OFP microbubbles were condensed into liquidfilled nanodroplets (i.e., PCCAs) [20]. Microbubble vials were cooled in an isopropanol/ $/ \mathrm{CO}_{2}$ bath maintained between -10 and $-13{ }^{\circ} \mathrm{C}$. Simultaneously, the headspace pressure of the vials was gradually increased through the addition of excess OFP gas until microbubble condensation was observed. Phase transition is visually apparent, as the initially opaque microbubble solution turns translucent when condensed into liquid-filled particles.

The size distribution and concentration of the PCCAs were characterized using a NanoSight NS500 (Malvern Instruments, Westborough, MA, USA) capable of detecting nanoparticles between 50 and $2000 \mathrm{~nm}$. PCCAs were diluted 3000-fold in HPLC-grade, $20 \mathrm{~nm}$ filtered water. Four, 30-s recordings were captured per sample to calculate an average size distribution and concentration for each sample. This procedure was repeated in triplicate for three separate vials of PCCAs and averaged to get a representative size distribution and concentration. The particles were characterized by a polydisperse size distribution, as in Fig. 1, with a mean size of $143 \pm 13 \mathrm{~nm}$ and concentration of $1.7 \pm 0.1 \times 10^{12}$ particles $/ \mathrm{mL}$ (see Additional file 1 for error estimation).

\section{Visualization of PCCA vaporization and secondary microbubble affects using optical microscopy and high-speed photography}

High-speed optical microscopy was used to detect PCCA vaporization following ultrasound stimulation using a previously described experimental setup [21, 22]. Briefly, an inverted microscope with a $100 \times$ water immersion objective (Olympus IX71, Center Valley, PA, USA) was interfaced with a high-speed camera (FastCam SA1.1, Photron USA, Inc., San Diego, CA, USA). The 


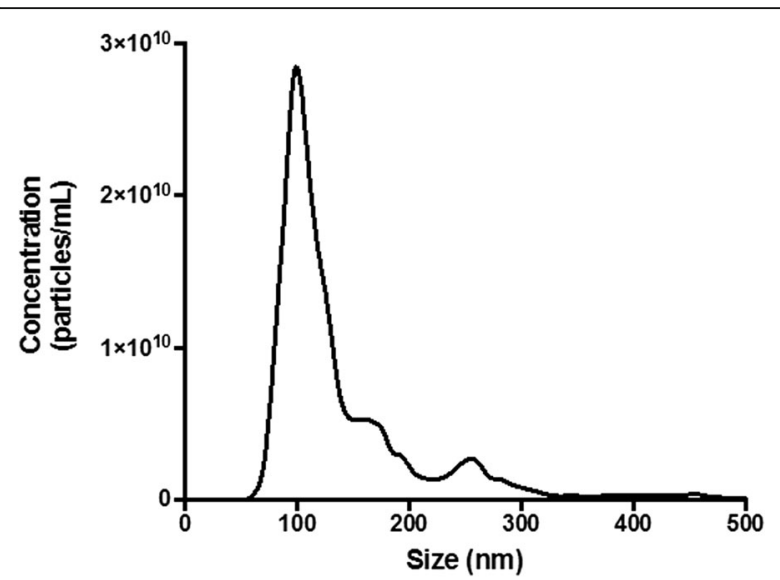

Fig. 1 Nanosight results for OFP-filled PCCAs ( $N=3$ vials). The mean particle size $( \pm$ SD) was found to be $140 \pm 10 \mathrm{~nm}$ and the average concentration $( \pm \mathrm{SD})$ was $1.7 \pm 0.1 \times 10^{12}$ particles $/ \mathrm{mL}$

objective was submerged in a temperature-controlled water bath fixed on top of the microscope. The water bath was filled with degassed water and held at $37{ }^{\circ} \mathrm{C}$. A solution of PCCAs diluted in PBS $(6.7 \% \quad v / v)$ was injected into a microcellulose tube $(200-\mu \mathrm{m}$ inner diameter) (Spectrum Labs, Inc., Rancho Dominguez, CA, USA) positioned over the optical focus. This injection was followed by a brief waiting period to allow the flowing particles to become nearly stationary. This enabled clear visualization of vaporization events as images become blurred when particles are flowing.

A $1.0-\mathrm{MHz}$ spherically focused piston transducer (diameter $=19 \mathrm{~mm}$, focal distance $=38 \mathrm{~mm}$, IL0106HP, Valpey Fisher Corp., Hopkinton, MA, USA) was submerged in the water bath and positioned such that the acoustic focus was aligned with the microcellulose tube at the optical focus as described previously [22]. Briefly, a calibrated needle hydrophone (HNA-0400, Onda Corp., Sunnyvale, CA, USA) was aligned with the microscope focus and used to subsequently align the focus of the transducer to that location. The hydrophone was then used to calibrate the pressure output of the transducer at various excitation voltages. The transducer was excited with sinusoidal pulses generated with an arbitrary waveform generator (AFG3021C, Tektronix, Inc., Beaverton, OR, USA) and amplified approximately $60 \mathrm{~dB}$ with a power amplifier (A500, ENI, Rochester, NY, USA). Following calibration, the hydrophone was replaced with a microcellulose tube, which was aligned with the microscope focus. In this way, we ensured that the plane of the tube visible in the optical focus was subjected to the calibrated acoustic pressures aligned to that location.

PCCAs flowing through the microcellulose tube were exposed to acoustic pulses with lengths of $5,10,20$, and 50 cycles and peak negative pressures of $125,300,600$,
1000 , and $2000 \mathrm{kPa}$ to observe the effect of pulse length and pressure on PCCA vaporization. In subsequent experiments, pre-vaporized PCCAs were stimulated with a second identical acoustic pulse to observe how ultrasound affected the generated microbubbles.

A synchronization pulse from the waveform generator was used to trigger the high-speed camera. Video recordings were set to begin just before the manually triggered ultrasound pulse such that vaporization or microbubble manipulations would be recorded in their entirety. A frame rate of 500 frames per second was employed. Images and videos were stored on a computer using proprietary camera software (PFV; Photron USA, Inc., San Diego, CA, USA) and analyzed using ImageJ (NIH, Bethesda, MD, USA).

\section{Detection of cavitation signals following PCCA vaporization}

Similar to the high-speed microscopy experiments, PCCA solutions were perfused through a microcellulose tube $(200 \mu \mathrm{L} / \mathrm{min})$ aligned with the focus of a $1.0-\mathrm{MHz}$ piston transducer. The transducer was calibrated at the focus using a needle hydrophone, and PCCAs were activated with sinusoidal ultrasound pulses using a pulse repetition frequency (PRF) of $5.0 \mathrm{~Hz}$, peak negative pressures ranging from 125 to $2000 \mathrm{kPa}$, and pulse lengths between 5 and 50 cycles. Three concentrations of PCCAs were tested: 0.067, 0.67, and 6.7\% $(v / v)$ in PBS. All conditions and concentrations were tested in triplicate using three independent vials of PCCAs. Control trials with a water-filled tube were used as a reference to estimate stable and inertial cavitation generated by the vaporized PCCAs.

To detect cavitation signals, a separate, spherically focused receive transducer $(7.5 \mathrm{MHz}$ center frequency, diameter $=19 \mathrm{~mm}$, focal distance $=50 \mathrm{~mm}) \quad(\mathrm{V} 321$, Panametrics, Inc., Waltham, MA, USA) was positioned perpendicular to the transmit transducer such that the microcellulose tube was aligned with both transducer foci. Signals from the receive transducer were acquired using a 14-bit analog to digital conversion (ADC) card with a sampling frequency of $100 \mathrm{MHz}$ (PDA14, Signatec, Corona, CA, USA) installed in a computer (Dell, Round Rock, TX, USA) running a custom acquisition program (LabVIEW, National Instruments Corp., Austin, TX, USA). A total of 50 individual signals were captured for each combination of pressure, pulse length, and PCCA concentration. These signals were saved and postprocessed using MATLAB (MathWorks Inc., Natick, MA, USA).

A custom MATLAB script was developed to quantify the energy of stable and inertial cavitation generated for each condition. First, a window from $50-110 \mu$ s referenced to the beginning of the acoustic pulse was applied 
to select the signal emitted by the PCCAs. The 50 individual RF signals from each exposure condition were converted into the frequency domain. Detection of the second harmonic component was used to estimate the stable cavitation level by filtering the data from 1.8 to 2.2 $\mathrm{MHz}$ (Butterworth filter, order 3). The broadband signal resulting from inertial cavitation was detected by filtering the signals from 5.25 to $7.75 \mathrm{MHz}$ (Butterworth filter, order 3 ) and by simultaneously excluding the harmonic components at 6 and $7 \mathrm{MHz}$. Finally, energies of these stable and inertial cavitation signals were calculated, averaged among the 50 individual signals for each condition, and normalized by the energy calculated for a water-filled tube exposed to the same acoustic conditions. This procedure was repeated for three independent vials of PCCAs. The average, normalized cavitation energies are reported with the inter-vial standard deviation.

\section{Cell culture}

Human pancreatic adenocarcinoma cells (PANC-1) were purchased from American Type Culture Collection (ATCC, VA, USA) and cultured in Dulbecco's Modified Eagle's Medium (DMEM) supplemented with $10 \%$ fetal bovine serum (FBS) and $1 \%$ penicillin-streptomycin (Sigma-Aldrich Co., MO, USA) at $37{ }^{\circ} \mathrm{C}$ and $5 \% \mathrm{CO}_{2}$ atmosphere. For all experiments, cells between passages 5 and 24 were used. Cells were harvested using trypsinEDTA (Sigma-Aldrich Co., MO, USA) and counted using a hemocytometer for use in sonoporation and viability experiments.

\section{Sonoporation of cells in suspension}

PANC- 1 cells $\left(1.0 \times 10^{6}\right.$ cells) were suspended in serumfree DMEM containing PCCAs $\left(8.5 \times 10^{8}\right.$ particles $)$ and propidium iodide (PI, $30 \mu \mathrm{M})$ (Sigma-Aldrich Co., MO, USA) for a final volume of $1.5 \mathrm{~mL}$. PI was used as a sonoporation indicator as it is impermeable to intact cell membranes. The cell suspension was added to a custom plastic cuvette with nearly acoustically transparent windows made of $20-\mu \mathrm{m}$ thick polyolefin film (Rajashrink, Roissy, France) as previously described by Escoffre et al. [23]. The cuvette was then held in a $37{ }^{\circ} \mathrm{C}$ degassed water bath with constant magnetic stirring and positioned $5 \mathrm{~cm}$ in front of the transducer for sonoporation treatment, as shown in Fig. 2.

To generate ultrasound pulses, a $1.0-\mathrm{MHz}$ unfocused piston transducer $($ diameter $=2.54 \mathrm{~cm}$, IL0108HP, Valpey Fisher Corp., Hopkinton, MA, USA) was excited by a sinusoidal arbitrary function generator signal (AFG3021C, Tektronix, Inc., Beaverton, OR, USA) amplified approximately $55 \mathrm{~dB}$ by an RF power amplifier (3100LA, ENI, Rochester, NY, USA). The pressure output of the transducer at various excitation voltages was characterized

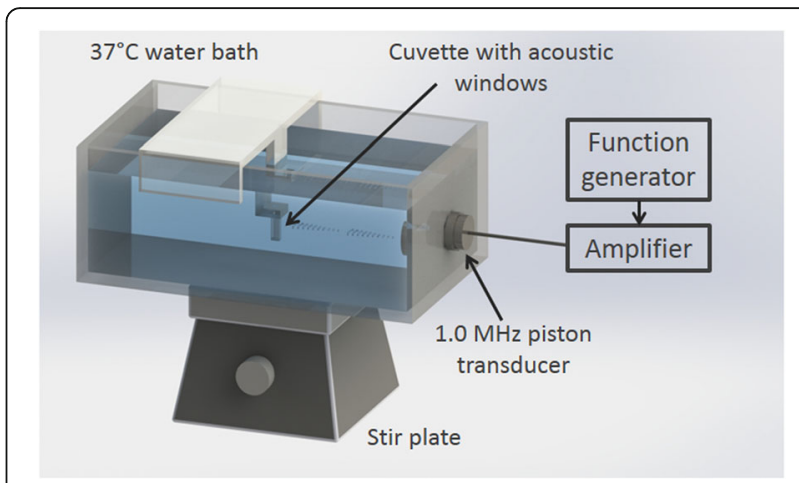

Fig. 2 Setup designed for the sonoporation of cells in suspension with PCCAs

using a calibrated needle hydrophone placed $5 \mathrm{~cm}$ in front of the transducer, matching the distance of the cuvette in sonoporation experiments. The cell suspensions were insonified for $30 \mathrm{~s}$ with peak negative pressures of 125 , 300 , or $600 \mathrm{kPa}$, pulse lengths of $5,10,20$, or 50 cycles, and a constant PRF of $5.0 \mathrm{kHz}$, as summarized in Table 1 . As controls, cells underwent (1) sham treatment (without PCCAs or ultrasound exposure) and (2) ultrasound-only treatment (without PCCAs) using the highest energy condition $-600 \mathrm{kPa}$ and 50 cycles.

Post-treatment, cells were transferred to plastic tubes and incubated at $37{ }^{\circ} \mathrm{C}$ for at least $15 \mathrm{~min}$ to ensure membrane resealing processes were completed prior to further manipulation of the cells [24]. Subsequently, the viability stain calcein-AM $(0.8 \mu \mathrm{M})$ (Thermo Fisher Scientific Inc., MA, USA) was added and the cells were allowed to incubate for at least an additional $30 \mathrm{~min}$ at $37{ }^{\circ} \mathrm{C}$. Cells were filtered through a $44-\mu \mathrm{m}$ nylon mesh (Component Supply Co., FL, USA) before being analyzed by flow cytometry. Cells showing both PI uptake and calcein-AM cleavage by flow cytometry were considered to be successfully sonoporated. These experiments were repeated in triplicate on independent days. All sonoporation conditions were also performed in triplicate without the addition of dye to monitor changes in autofluorescence due to treatment.

\section{Assessment of sonoporation efficiency by flow cytometry} Flow cytometry was used to quantify the number of sonoporated cells for each treatment group, i.e., those

Table 1 Experimental and control conditions for sonoportion

\begin{tabular}{lllll}
\hline Conditions & PCCAs (Y/N) & Cycles (\#) & PRF $(\mathrm{kHz})$ & Pressure $(\mathrm{kPa})$ \\
\hline $1-4$ & $\mathrm{Y}$ & $5,10,20,50$ & 5 & 125 \\
$5-8$ & $\mathrm{Y}$ & $5,10,20,50$ & 5 & 300 \\
$9-12$ & $\mathrm{Y}$ & $5,10,20,50$ & 5 & 600 \\
US only control & $\mathrm{N}$ & 50 & 5 & 600 \\
Sham control & $\mathrm{N}$ & $\mathrm{NA}$ & $\mathrm{NA}$ & $\mathrm{NA}$ \\
\hline
\end{tabular}


cells displaying both PI uptake (permeabilization) and calcein-AM cleavage (viability). An LSRFortessa cytometer equipped with 561- and 488-nm excitation lasers (Becton Dickinson, Franklin Lakes, NJ, USA) was used for acquisition, and 30,000 events were recorded for each sonoporation treatment. For further details regarding acquisition settings, please see Additional file 1: Table S2.

The gating strategy employed to isolate sonoporated cells is described in full in Additional file 1: Information section and displayed in Additional file 2: Figure S1. Briefly, singlet cells were isolated from debris and doublet cells through initial gating steps. The viability of the chosen cell population was then confirmed by calcein fluorescence. Curly quadrant gates were applied to the calcein vs. PI fluorescence dot plots, with thresholds determined such that unstained control cells would be classified as both calcein and PI negative. The percent of cells in quadrant two (calcein and PI positive) was taken to be the sonoporation efficiency (i.e., percent of viable cells that were sonoporated). All data analysis was performed using FlowJo Data Analysis Software (FlowJo, LLC., Ashland, OR, USA).

\section{Assessing viability post-sonoporation treatment}

Through our flow cytometry experiments, we found that dead cells and cellular debris were characterized by elevated autofluorescence in the calcein (viability) channel (data not shown). Therefore, we were unable to accurately quantify cell viability based on the flow cytometry results alone. As such, we performed an additional cell viability assay. Cells suspended in serum-supplemented DMEM were subjected to the sonoporation protocol as described above without the addition of PI or calceinAM. Following treatment, $1.0 \times 10^{5}$ cells per treatment group were transferred to 24-well plates and allowed to incubate for $24 \mathrm{~h}$ at $37{ }^{\circ} \mathrm{C}$ and $5 \% \mathrm{CO}_{2}$ atmosphere. Subsequently, cell viability was assessed using a resazurinbased toxicology assay according to the manufacturer's protocol (Sigma-Aldrich Co., MO, USA).

Briefly, a volume of resazurin dye equal to $10 \%$ of the culture media was added to the cells and allowed to incubate for $3 \mathrm{~h}$. A $200-\mu \mathrm{L}$ sample from each culture well was then transferred to a 96-well plate for analysis. The fluorescence increase at $590 \mathrm{~nm}\left(\mathrm{~F}_{590}\right)$ due to reduction of the resazurin dye by viable cells was detected using a plate reader (Synergy 2, BioTek Instrument, Inc., Winooski, VT, USA) with excitation and emission filters of 530/25 nm and 590/35 nm, respectively. The fluorescence intensity of a blank sample containing complete media but no cells was subtracted from that of each sample. Cell viability was then calculated as the percent resazurin reduction of the sham control. Viability experiments were repeated in triplicate on independent days.

\section{Statistical analyses}

All statistical analyses were performed in GraphPad Prism 7 (GraphPad Software, Inc., La Jolla, CA, USA), and data are presented as average \pm standard deviation throughout this work. Sonoporation efficiencies and cell viabilities were compared among treatment groups using one-way ANOVA followed by Dunnett's multiple comparison testing on significant results. Each treatment group was compared to the sham control, and $p$ values of $<0.05$ were considered statistically significant. Pearson correlation coefficients $(r)$ were computed in GraphPad Prism 7 to analyze the correlation between (1) sonoporation efficiency and stable cavitation and (2) sonoporation efficiency and inertial cavitation. Correlations were considered statistically significant if the two-tailed $p$ values were $<0.05$.

\section{Results}

\section{Detection of PCCA vaporization and subsequent} cavitation signals

Through optical high-speed microscopy and the detection of cavitation signals, we investigated the effect of acoustic pulse length and peak negative pressure on PCCA vaporization (a.k.a. acoustic droplet vaporization $(\mathrm{ADV}))$ and the behavior of resultant microbubbles. At a frequency of $1.0 \mathrm{MHz}$, we found that our PCCAs undergo $\mathrm{ADV}$ at and above peak negative pressures of $300 \mathrm{kPa}$ but never at or below $125 \mathrm{kPa}$, regardless of pulse length. This is consistent with previous reports demonstrating that ADV is a pressure-dependent, threshold phenomenon that is independent of pulse length when short, microsecond pulses are used [25, 26]. Representative photos showing PCCAs before and after ultrasound stimulation above and below the activation threshold are displayed in Fig. 3a. Note that $300 \mathrm{kPa}$ does not represent an absolute pressure threshold for vaporization; rather, we conclude that the vaporization threshold is between 125 and $300 \mathrm{kPa}$ under the conditions studied.

While pulse length did not effect whether or not vaporization would occur, it did influence the behavior of resultant microbubbles. When generated microbubbles were stimulated with a second ultrasound pulse, microbubble destruction occurred on a continuum. No destruction was observed with low-pressure pulses $(300 \mathrm{kPa})$ and complete destruction of all microbubbles in the field of view occurred with long pulses (20 and 50 cycles $)$ at high pressure $(1000$ and $2000 \mathrm{kPa})$ (Fig. 3b).

Microbubble sizes were estimated from the captured images. Microbubbles generated from ADV at $300 \mathrm{kPa}$ were polydisperse and ranged in size between 2 and $10 \mu \mathrm{m}$. When the peak negative pressure was increased to $600 \mathrm{kPa}$ and above, generated microbubbles were 

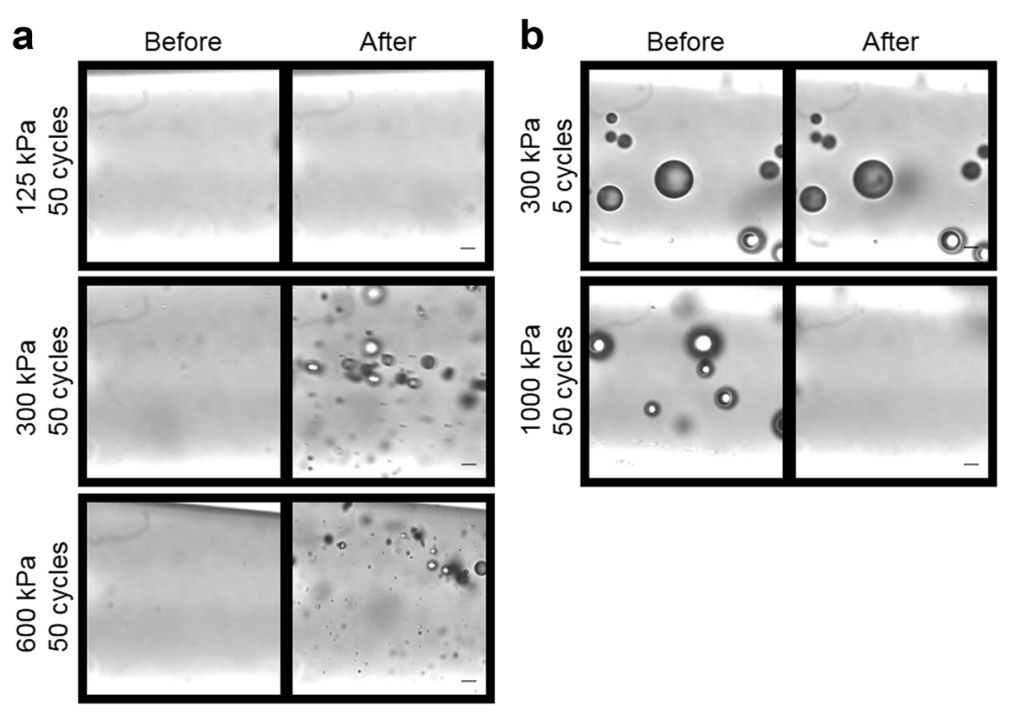

Fig. 3 Observation of PCCA vaporization and secondary microbubble effects using high-speed photography. Representative photos are displayed of PCCAs or resultant microbubbles before and after ultrasound stimulation (1.0 MHz center frequency). a The nanoscale, liquid-filled PCCAs are difficult to observe before vaporization. A peak negative pressure of $125 \mathrm{kPa}$ is not sufficient to vaporize the PCCAs (top). With a peak negative pressure of $300 \mathrm{kPa}$, efficient vaporization of the PCCAs into microbubbles is observed (bottom). b Secondary effects are observed when generated microbubbles are subjected to a second acoustic pulse. At $300 \mathrm{kPa}$ and 5 cycles, the second acoustic pulse appears to have no affect on the generated microbubbles (top). With high acoustic energies, complete microbubble destruction is observed (bottom). Scale bar $=10 \mu \mathrm{m}$. a Vaporization b Secondary effects

observed in the 1- to $10-\mu \mathrm{m}$ range; however, we note an increase in the number of small $(\sim 1 \mu \mathrm{m})$ microbubbles present. This is consistent with previous reports from our laboratory detailing the dependence of generated microbubble size on various acoustic parameters, including peak negative pressure [27]. The shift towards smaller resultant microbubbles with increased pressure is due to the inverse relationship between vaporization threshold and PCCA size [27, 28]. When generated microbubbles were allowed to rest before being subjected to a second acoustic pulse (Fig. 3b), we noticed microbubble sizes shift to be larger (approximately 3-20 $\mu \mathrm{m}$ ). This is likely due to coalescence of the generated microbubbles.
The generation of stable and inertial cavitation signals depended on peak negative pressure and pulse length. Very little stable and no inertial cavitation was observed at pressures of $125 \mathrm{kPa}$ regardless of pulse length; the slight stable cavitation may be due to oscillations of microbubbles that arose from spontaneous vaporization. Cavitation energy was observed from 300 to $2000 \mathrm{kPa}$, with very little cavitation achieved with peak negative pressures of $300 \mathrm{kPa}$ and short (5 and 10 cycle) pulse lengths (Fig. 4). The amount of stable cavitation produced reached a plateau between 24 and $29 \mathrm{~dB}$ for 50 cycle pulses with pressures between 300 and $2000 \mathrm{kPa}$. Alternatively, inertial cavitation continued to increase with increasing pressure. Interestingly, the
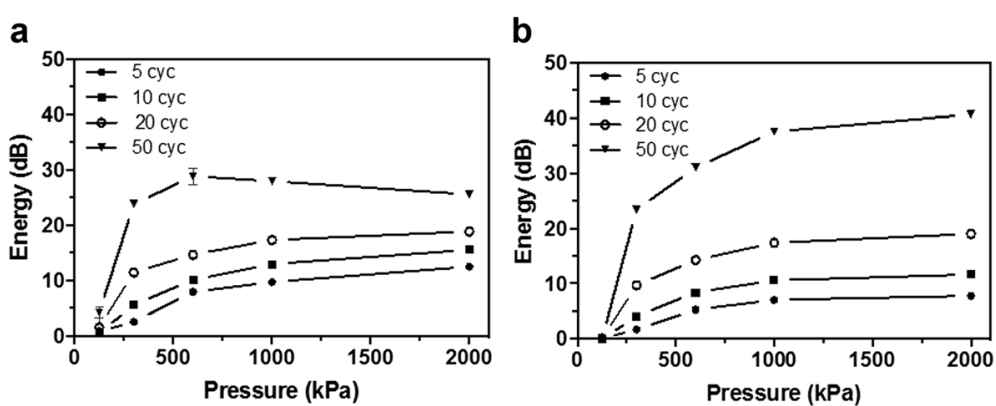

Fig. 4 Quantification of the $\mathbf{a}$ stable and $\mathbf{b}$ inertial cavitation energy generated by PCCAs subjected to ultrasound of various peak negative pressures and pulse lengths. Note: while error bars (SD) are plotted, they are not visible on all data points due to their small size. a Stable Cavitation Detection b Inertial Caviatation Detection 
concentration of PCCAs did not significantly influence the amount of stable or inertial cavitation detected (data not shown), and obtained graphs for all tested concentrations were nearly identical to the one presented in Fig. 4 for $0.67 \%(v / v)$ PCCAs in PBS.

\section{Sonoporation efficiency}

Flow cytometry was used to analyze the effect of acoustic pressure and pulse length on PCCA-facilitated PI uptake through sonoporation. Dead cells were discarded from the analysis through an initial gating step to remove cellular debris (Additional file 2: Figure S1). The viability of remaining cells was confirmed using calceinAM staining. While the viability of gated cells was near $100 \%$ for all conditions, the percentage of cellular debris was observed to increase with increasing acoustic energy, implying elevated cell death.

Sonoporation efficiency, the percent of viable cells displaying PI fluorescence, was quantified as the percent of cells in quadrant 2, as shown in Fig. 5. A small percentage of cells $(2-4.5 \%)$ appeared in quadrant two for the sham control, likely due to the prolonged exposure of cells to PI. This was defined as the false positive rate and was subtracted from the sonoporation efficiency of all other treatment groups. The autofluorescence analysis demonstrated slight spreading of unstained cell populations along the PI axis due to ultrasound treatment with PCCAs (Additional file 3: Figure S2). The average percent of cells classified as PI positive due to autofluorescence never exceeded $2 \%$, but these values were subtracted from the final sonoporation efficiencies of all groups.

Statistically significant elevation in PI uptake was observed at $300 \mathrm{kPa}$ with pulse lengths of 20 and 50 cycles and at $600 \mathrm{kPa}$ with 5-50 cycle pulse lengths compared to the sham control. Sonoporation efficiency increased with peak negative pressure and pulse length, reaching a maximum of $36 \pm 4 \%$ at $600 \mathrm{kPa}$ and 50 cycles (Fig. 6). As expected, we did not observe sonoporation below the vaporization threshold of the PCCAs (i.e., at $125 \mathrm{kPa}$ ) or when cells were insonified in the absence of PCCAs.

\section{Cell viability $24 \mathrm{~h}$ post-treatment}

To test the effect of sonoporation treatment on cell viability, cells were treated using a protocol identical to that employed for sonoporation but without the addition of
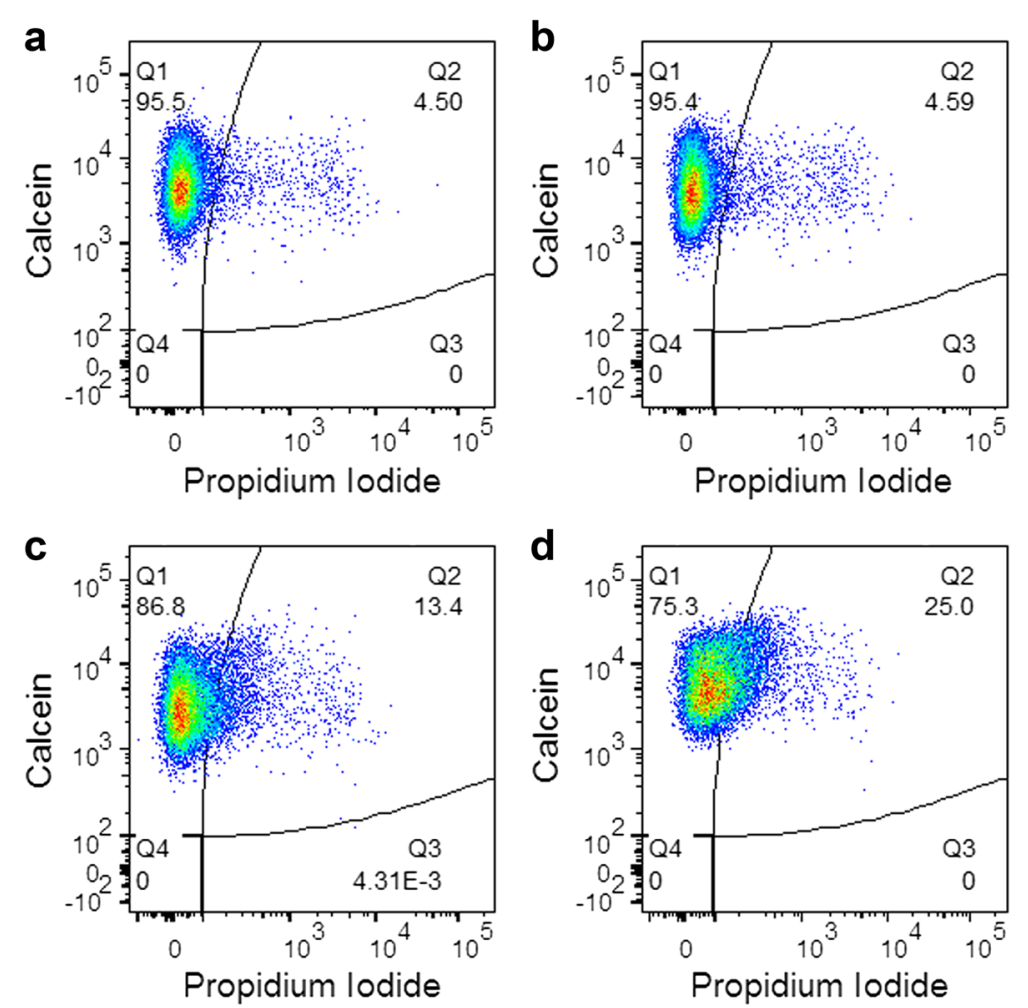

Fig. 5 Representative flow cytometry dot plots used to quantify sonoporation efficiency. Cells were classified as sonoporated if they showed calcein fluorescence (viability) and uptake propidium iodide (membrane permeability). These cells appear in quadrant 2 (Q2) in the dot plots. a The small percentage of cells that appear in Q2 for the sham treatment group was defined as the false positive rate and was subtracted from the percent of cells in Q2 for all treatment groups. $\mathbf{b}$ Ultrasound exposure below the PCCA activation threshold did not result in sonoporation. c, d Sonoporation is observed above the PCCA activation threshold and increases with increasing pressure. a Sham Control b $125 \mathrm{kPa}-20 \mathrm{cycles}$ c $300 \mathrm{kPa}-20$ cycles $\mathbf{d} 600 \mathrm{kPa}-20$ cycles 


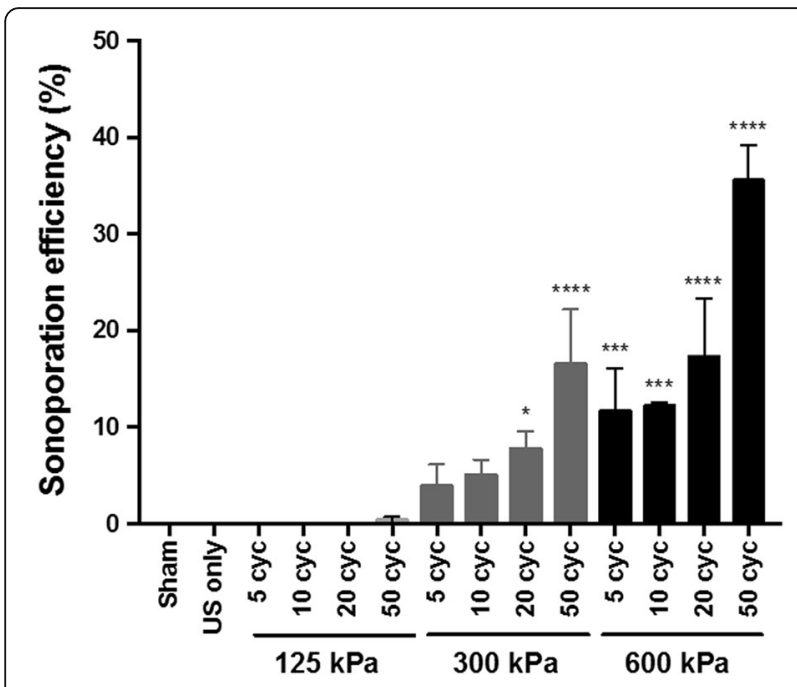

Fig. 6 Sonoporation efficiency of PANC-1 cells at various acoustic pressures and pulse lengths. As expected, we do not observe sonoporation below the vaporization threshold of the PCCAs (i.e., at $125 \mathrm{kPa}$ ) or when cells are insonified in the absence of PCCAs (US alone). One-way ANOVA was used followed by Dunnett's multiple comparisons test compare each treatment to the sham control. ${ }^{*} p \leq 0.05,{ }^{* *} p \leq 0.001,{ }^{* * * *} p \leq 0.0001$

PI or calcein-AM. Twenty-four hours post-treatment, viability was assessed using a resazurin-based metabolic assay. Ultrasound exposure in the absence of PCCAs did not affect cell viability. Furthermore, the PCCAs themselves did not have a toxic effect on cells as evidenced by the high viability in treatment groups below the PCCA activation threshold (i.e., $125 \mathrm{kPa}$ treatment groups). We did observe decreasing cell viability with increasing cycle number and pressure above the activation threshold. In general, fairly high viability was recorded for those cells treated with $300 \mathrm{kPa}$ ultrasound of various pulse lengths $(84 \pm 7 \%-94 \pm 7 \%$ viability) and cells treated with $600 \mathrm{kPa}$ ultrasound with pulse lengths between 5 and 20 cycles ( $85 \pm 12 \%-93 \pm 6 \%$ ) (Fig. 7). A statistically significant drop in viability $(70 \pm 5 \%)$ was observed in cells treated with $600 \mathrm{kPa}$ and 50 cycles compared to sham treated cells.

\section{Discussion}

\section{Acoustic or temperature-induced droplet vaporization} can be achieved without membrane perforation or impaired cell viability

Our PCCAs are comprised of a very low-boiling point PFC and undergo some spontaneous vaporization when incubated at $37^{\circ} \mathrm{C}$. Therefore, cells incubated with PCCAs and exposed to ultrasound below the activation threshold (i.e., at $125 \mathrm{kPa}$ ) felt the effects of temperature-induced vaporization alone. The membrane permeability and viability of cells treated in this way was unaltered. Additionally, cells treated with PCCAs at $300 \mathrm{kPa}$ with pulse lengths of

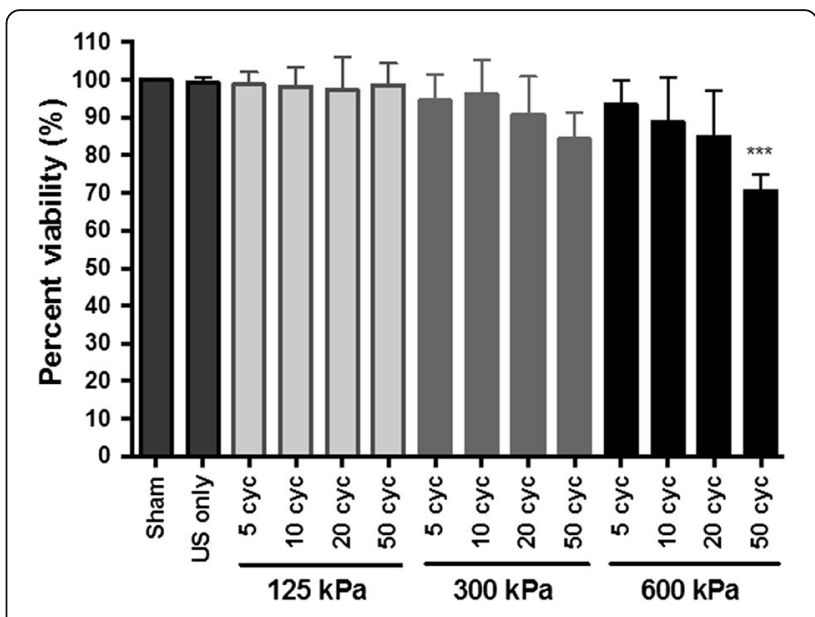

Fig. 7 Cell viability 24 h post-sonoporation treatment. Here, we observe decreasing cell viability with increasing pulse length and pressure. As expected, ultrasound exposure in the absence of PCCAs does not affect cell viability. Furthermore, the PCCAs themselves did not have a toxic effect on cells as evidenced by the high viability in treatment groups below the PCCA activation threshold (i.e., $125 \mathrm{kPa}$ groups). One-way ANOVA was used followed by Dunnett's multiple comparisons test to compare each treatment to the sham control. ${ }^{* *} p \leq 0.001$

5 or 10 cycles demonstrated insignificant sonoporation efficiencies and no change in cell viability. These cells were exposed to acoustic droplet vaporization but minimal cavitation of the resultant microbubbles. These data indicate that vaporization events do not affect cellular membrane permeability or cause any detrimental cellular bioeffects.

This is in contrast to the bioeffects observed following the vaporization of micron-sized, DDFP-filled PCCAs used for vascular occlusion. Seda, et al. have demonstrated that vaporization of DDFP-filled droplets results in extensive cell death even when using acoustic parameters designed to minimize secondary mechanical effects from the resultant bubbles [29]. Differences in experimental setups (cells treated in adherent culture vs. in suspension) and size distributions of PCCAs $(1.6 \pm 0.5 \mu \mathrm{m}$ vs. $143 \pm 13 \mathrm{~nm}$ mean size) make it difficult to directly compare these results. However, the difference in severity of bioeffects observed is likely due to the difference in pressure required to vaporize the PCCAs. Rarefactional pressures of at least $6 \mathrm{MPa}$ were required for vaporization of DDFP-filled PCCAs, while $300 \mathrm{kPa}$ was sufficient for vaporization of our PCCAs. By using a highly volatile formulation with lower pressure requirements for ADV, we can safely induce vaporization without immediately and irreparably damaging surrounding cells.

\section{PCCA-induced sonoporation is correlated with stable and inertial cavitation}

Sonoporation efficiency was found to be significantly and positively correlated with both stable $(r=0.9352$, 
$p<0.0001)$ and inertial $(r=0.9456, p<0.0001)$ cavitation (Fig. 8). While it is difficult to ascertain a cavitation threshold for sonoporation from these data, we note that all statistically significant sonoporation treatments were associated with stable cavitation energies greater than $7.9 \mathrm{~dB}$ and inertial cavitation energies greater than $5.2 \mathrm{~dB}$. This study was not designed to elucidate the mechanisms driving PCCA-mediated sonoporation, but our data suggest that the mechanical effects due to microbubbleultrasound interactions are necessary for significant sonoporation. Therefore, it is likely that the same mechanisms that drive conventional microbubble-mediated sonoporation also drive PCCA-mediated sonoporation.

The peak sonoporation efficiency we achieved $(36 \%)$ is similar to what has previously been reported for microbubble sonoporation (28-39\% efficiency) [6, 30, 31], albeit with lower cell viability $(70 \%$ viability for PCCA sonoporation vs. $90-96 \%$ viability for $\mathrm{MB}$ sonoporation $[6,30,31])$. However, these microbubble sonoporation studies employ unique strategies to increase sonoporation efficiency and minimize cell death, making it difficult to make direct comparisons. For example, McLaughlan et al. achieved their highest viable sonoporation using a combination of (1) targeted microbubbles that increase cell-microbubble interactions and (2) chirp frequency excitation to maximize the response of their polydisperse microbubbles [6]. Song et al. found that using monodisperse $2.0-\mu \mathrm{m}$ microbubbles resulted in the highest sonoporation and viability after a single ultrasound treatment [31]. We believe that with further optimization of our PCCA-mediated sonoporation methods, we will be able to match the sonoporation efficiencies and viabilities achieved with microbubbles. Future studies will be designed to apply the aforementioned techniques developed by the microbubble sonoporation community to PCCA-mediated sonoporation.

\section{Conclusions}

In conclusion, our data show that low-boiling point PCCAs are capable of inducing sonoporation without causing detrimental cellular bioeffects in vitro. Furthermore, the low pressure required to activate such PCCAs allows us to finetune the severity of cellular bioeffects simply by modifying pulse length. This provides flexibility in future applications imaginable and allows for acoustic droplet vaporization to be achieved safely and with existing diagnostic imaging hardware. Here we demonstrate the ability to cause (1) vaporization with no cellular damage-ideal for diagnostic imaging applications, (2) reversible sonoporation-desirable for therapeutic applications such as drug or gene delivery where cell death is to be avoided, or (3) irreversible sonoporation-useful in augmenting tumor killing through high-intensity focused ultrasound treatment.

A limitation of this study is that we did not control for differences in PCCA vaporization efficiency at each acoustic condition. In other words, more bubbles were likely generated using the highest energy conditions compared to the lowest energy conditions as a constant PCCA concentration was used throughout. This makes it difficult to draw conclusions about sonoporation mechanism and parameter optimization. The increases in sonoporation efficiency with increasing pressure and pulse length may have been due to (1) increased cavitation and associated mechanical effects, (2) increased concentration of generated microbubbles, or (3) a combination thereof. Future studies will be designed to quantify the vaporization efficiency of PCCAs at each acoustic condition to allow for concentration matching
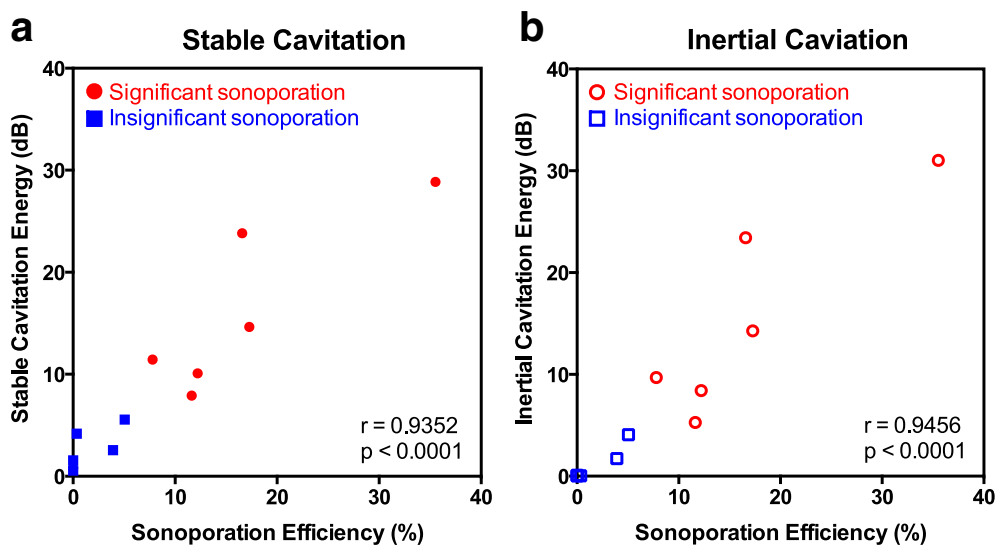

Fig. 8 We observe a strong, positive correlation between sonoporation efficiency and a stable cavitation and $\mathbf{b}$ inertial cavitation. The Pearson correlation coefficients for sonoporation efficiency vs. stable and inertial cavitation are 0.9352 and 0.9456 , respectively, and both correlations are statistically significant with $p$ values $<0.0001$. Data points corresponding to statistically significant sonoporation efficiencies (compared to sham control) are shown in red circles, while data points corresponding to statistically insignificant sonoporation efficiencies are shown in blue squares. a Stable Cavitation b Inertial Caviation 
of generated microbubbles. Other important parameters to consider are contrast agent size distribution, ultrasound exposure duration, and center frequency. Future studies will be designed to optimize these parameters and provide a thorough comparison between the sonoporation potential of microbubbles, low-boiling point PCCAs, and high-boiling point PCCAs.

One of the main advantages of using PCCAs for reversible sonoporation compared to microbubbles is the potential for their extravasation from a tumor's leaky vasculature. While we note that the mean size of our PCCAs is smaller than the pore sizes in many permeable tumor lines $(200-1.2 \mu \mathrm{m})$ [32], the extravasation and accumulation of our particles in tumors has yet to be confirmed. Studies are currently ongoing towards this end. Nevertheless, our data warrant further investigation into the use of PCCAs to induce extravascular sonoporation in vivo for the purpose of enhancing local drug or gene delivery, particularly within solid tumors.

\section{Additional files}

Additional file 1: S.1. Error estimation for PCCA size and concentration. Table S1. Error estimation for PCCA size and concentration. S.2. Details regarding flow cytometry analysis. Table S2. Detector voltages used for all flow cytometry acquisitions. (DOCX $25 \mathrm{~kb}$ )

Additional file 2: Figure S1. Gating hierarchy used for sonoporation detection. (TIF $114 \mathrm{~kb}$ )

Additional file 3: Figure S2. A) Gating hierarchy for detecting autofluorescence in treated cells. First, cells were isolated from debris using FSC-A vs. SSC-A. Second, singlet cells were isolated using FSC-A vs. FSC-H. Third, quadrant gates were drawn identical to those used for quantifying sonoporation. B) Representative dot plots demonstrating slight spreading (autofluorescence) of cells treated with ultrasound and PCCAs. (TIF $123 \mathrm{~kb}$ )

\section{Abbreviations}

ADV: Acoustic droplet vaporization; DDFP: Dodecafluoropentane; OFP: Octafluoropropane; PCCA: Phase-change contrast agent; PI: Propidium iodide; US: Ultrasound

\section{Acknowledgements \\ The authors would like to thank Dr. James Tsuruta for the assistance with microbubble formulations and Dr. Russel Mumper for the use of his fluorescence plate reader. \\ All flow cytometry experiments were performed through the UNC Flow Cytometry Core Facility, and the authors appreciate the technical support and advice from Core staff, especially Dr. Nancy Fisher, Janet Dow, and Felicia Heyward.}

\section{Funding}

This research was supported by a Drugs, Devices and Diagnostics Development (4D) grant (\#4DR21402) supplied by The North Carolina Translational and Clinical Sciences (NC TraCS) Institute at The University of North Carolina at Chapel Hill. This funding agency did not participate in the design, analysis, or interpretation of any experiments presented here or in the preparation of this manuscript.

The UNC Flow Cytometry Core Facility is supported in part by P30 CA016086 Cancer Center Core Support Grant to the UNC Lineberger Comprehensive Cancer Center.

Research reported in this publication was supported by the Center for AIDS Research award number 5P30AI050410. The content is solely the responsibility of the authors and does not necessarily represent the official views of the National Institutes of Health.

C. B. Arena was supported by a grant from the National Institute of General Medical Sciences, division of Training, Workforce Development, and Diversity under the Institutional Research and Academic Career Development Award, grant \#K12-GM000678.

A. Novell wishes to thank the foundation ARC (no SAE20130606511) for the financial support.

P. A. Dayton declares that he is a co-founder and equity holder in Nanosonic Bioreagents, LLC, and also an inventor on patents licensed by Nanosonic Bioreagents which describe the low-boling point phase change contrast agents decribed here.

\section{Availability of data and materials}

Data from this work are not available through a public repository but can be obtained from the authors directly within 3 years of the publication date.

\section{Author contributions}

SMF fabricated the contrast agents, performed the optical microscopy and all cell-based experiments, and performed the flow cytometry analysis. She also analyzed and interpreted the results from these experiments and was the major contributor in writing this manuscript. AN was instrumental in analyzing the cavitation detection data and provided assistance in designing the sonoporation setup and executing the sonoporation experiments. YY assisted with the experimental design and provided advice regarding the preparation of this manuscript. PAD provided essential support and guidance regarding the experimental design and data interpretation. CBA assisted with the experimental design and execution throughout this work, assisted in the interpretation of results, and provided advice regarding the preparation of this manuscript. All authors read and approved the final manuscript.

\section{Competing interests}

P.A. Dayton declares that he is a co-inventor on a patent describing the formulation of low-boiling point perfluorocarbon agents and a co-founder of Nanosonic Bioreagents, LLC, a company which has licensed this patent and manufactures perfluorocarbon nanodroplets for commercial sale. All the other authors declare that they have no competing interests.

\section{Consent for publication}

Not applicable.

Ethics approval and consent to participate Not applicable.

\section{Author details}

${ }^{1}$ Eshelman School of Pharmacy, University of North Carolina Chapel Hill, Chapel Hill, NC, USA. ${ }^{2}$ Joint Department of Biomedical Engineering, University of North Carolina Chapel Hill and North Carolina State University, Chapel Hill, NC, USA. ${ }^{3}$ FIT BEST Laboratory, Chemical, Biological and Bioengineering Department, North Carolina A\&T State University, Greensboro, NC, USA. ${ }^{4}$ Laboratory for Therapeutic Directed Energy, Department of Physics, Elon University, Elon, NC, USA.

Received: 9 July 2016 Accepted: 6 January 2017

Published online: 24 January 2017

\section{References}

1. Lentacker I, De Cock I, Deckers R, De Smedt SC, Moonen CT. Understanding ultrasound induced sonoporation: definitions and underlying mechanisms. Adv Drug Deliv Rev. 2014;72:49-64.

2. Yu H, Xu L. Cell experimental studies on sonoporation: state of the art and remaining problems. J Control Release. 2014;174:151-60.

3. De Cock I, Zagato E, Braeckmans K, Luan Y, de Jong N, De Smedt SC, Lentacker I. Ultrasound and microbubble mediated drug delivery: acoustic pressure as determinant for uptake via membrane pores or endocytosis. J Control Release. 2015;197:20-8.

4. Fan Z, Kumon RE, Deng CX. Mechanisms of microbubble-facilitated sonoporation for drug and gene delivery. Ther Deliv. 2014;5:467-86.

5. Kivinen J, Togtema M, Mulzer G, Choi J, Zehbe I, Curiel L, Pichardo S. Sonoporation efficacy on SiHa cells in vitro at raised bath temperatures- 
experimental validation of a prototype sonoporation device. J Ther Ultrasound. 2015;3:19.

6. McLaughlan J, Ingram N, Smith PR, Harput S, Coletta PL, Evans S, Freear S. Increasing the sonoporation efficiency of targeted polydisperse microbubble populations using chirp excitation. IEEE Trans Ultrason Ferroelectr Freq Control. 2013;60:2511-20.

7. Ohl C-D, Arora M, Ikink R, de Jong N, Versluis M, Delius M, Lohse D. Sonoporation from jetting cavitation bubbles. Biophys J. 2006;91:4285-95.

8. Sirsi S, Borden M. Microbubble compositions, properties and biomedical applications. Bubble Sci Eng Technol. 2009;1:3-17.

9. Skachkov I, Luan Y, van der Steen AF, de Jong N, Kooiman K. Targeted microbubble mediated sonoporation of endothelial cells in vivo. IEEE Trans Ultrason Ferroelectr Freq Control. 2014;61:1661-7.

10. Downs ME, Buch A, Karakatsani ME, Konofagou EE, Ferrera VP. Blood-brain barrier opening in behaving non-human primates via focused ultrasound with systemically administered microbubbles. Sci Rep. 2015;5:15076.

11. Wu SY, Chen CC, Tung YS, Olumolade OO, Konofagou EE. Effects of the microbubble shell physicochemical properties on ultrasound-mediated drug delivery to the brain. J Control Release. 2015;212:30-40.

12. Sheeran PS, Rojas JD, Puett C, Hjelmquist J, Arena CB, Dayton PA. Contrastenhanced ultrasound imaging and in vivo circulatory kinetics with low-boilingpoint nanoscale phase-change perfluorocarbon agents. Ultrasound Med Biol. 2015:41:814-31.

13. Rapoport N, Nam K-H, Gupta R, Gao Z, Mohan P, Payne A, Todd N, Liu X, Kim T, Shea J, et al. Ultrasound-mediated tumor imaging and nanotherapy using drug loaded, block copolymer stabilized perfluorocarbon nanoemulsions. J Control Release. 2011;153:4-15.

14. Williams R, Wright C, Cherin E, Reznik N, Lee M, Gorelikov I, Foster FS, Matsuura N, Burns PN. Characterization of submicron phase-change perfluorocarbon droplets for extravascular ultrasound imaging of cancer. Ultrasound Med Biol. 2013:39:475-89.

15. Burgess MT, Porter TM. Acoustic cavitation-mediated delivery of small interfering ribonucleic acids with phase-shift nano-emulsions. Ultrasound Med Biol. 2015;41:2191-201.

16. Fabiilli ML, Haworth KJ, Sebastian IE, Kripfgans OD, Carson PL, Fowlkes JB. Delivery of chlorambucil using an acoustically-triggered perfluoropentane emulsion. Ultrasound Med Biol. 2010;36:1364-75.

17. Gao D, Xu M, Cao Z, Gao J, Chen Y, Li Y, Yang Z, Xie X, Jiang Q, Wang W, Liu J. Ultrasound-triggered phase-transition cationic nanodroplets for enhanced gene delivery. ACS Appl Mater Interfaces. 2015:7:13524-37.

18. Liu WW, Liu SW, Liou YR, Wu YH, Yang YC, Wang CR, Li PC. Nanodropletvaporization-assisted sonoporation for highly effective delivery of photothermal treatment. Sci Rep. 2016;6:24753.

19. Qin $P, X u L$, Han $T, D u L, Y u A C$. Effect of non-acoustic parameters on heterogeneous sonoporation mediated by single-pulse ultrasound and microbubbles. Ultrason Sonochem. 2016;31:107-15.

20. Sheeran PS, Luois SH, Mullin LB, Matsunaga TO, Dayton PA. Design of ultrasonically-activatable nanoparticles using low boiling point perfluorocarbons. Biomaterials. 2012;33:3262-9.

21. Sheeran PS, Luois S, Dayton PA, Matsunaga TO. Formulation and acoustic studies of a new phase-shift agent for diagnostic and therapeutic ultrasound. Langmuir. 2011;27:10412-20.

22. Sheeran PS, Wong VP, Luois S, McFarland RJ, Ross WD, Feingold S, Matsunaga TO, Dayton PA. Decafluorobutane as a phase-change contrast agent for low-energy extravascular ultrasonic imaging. Ultrasound Med Biol. 2011;37:1518-30.

23. Escoffre JM, Novell A, de Smet M, Bouakaz A. Focused ultrasound mediated drug delivery from temperature-sensitive liposomes: in-vitro characterization and validation. Phys Med Biol. 2013;58:8135-51.

24. Hu Y, Wan JM, Yu AC. Membrane perforation and recovery dynamics in microbubble-mediated sonoporation. Ultrasound Med Biol. 2013;39:2393-405.

25. Fabiilli ML, Haworth KJ, Fakhri NH, Kripfgans OD, Carson PL, Fowlkes JB. The role of inertial cavitation in acoustic droplet vaporization. IEEE Trans Ultrason Ferroelectr Freq Control. 2009;56:1006-17.

26. Lo AH, Kripfgans OD, Carson PL, Rothman ED, Fowlkes JB. Acoustic droplet vaporization threshold: effects of pulse duration and contrast agent. IEEE Trans Ultrason Ferroelectr Freq Control. 2007;54:933-46.

27. Sheeran PS, Matsunaga TO, Dayton PA. Phase-transition thresholds and vaporization phenomena for ultrasound phase-change nanoemulsions assessed via high-speed optical microscopy. Phys Med Biol. 2013;58:4513-34.
28. Sheeran PS, Dayton PA. Phase-change contrast agents for imaging and therapy. Curr Pharm Des. 2012;18:2152-65.

29. Seda R, Li DS, Fowlkes JB, Bull JL. Characterization of bioeffects on endothelial cells under acoustic droplet vaporization. Ultrasound Med Biol. 2015;41:3241-52.

30. Karshafian R, Bevan PD, Williams R, Samac S, Burns PN. Sonoporation by ultrasound-activated microbubble contrast agents: effect of acoustic exposure parameters on cell membrane permeability and cell viability. Ultrasound Med Biol. 2009:35:847-60.

31. Song KH, Fan AC, Brlansky JT, Trudeau T, Gutierrez-Hartmann A, Calvisi ML, Borden MA. High efficiency molecular delivery with sequential low-energy sonoporation bursts. Theranostics. 2015;5:1419-27.

32. Hobbs SK, Monsky WL, Yuan F, Roberts WG, Griffith L, Torchilin VP, Jain RK Regulation of transport pathways in tumor vessels: role of tumor type and microenvironment. Proc Natl Acad Sci U S A. 1998:95:4607-12.

\section{Submit your next manuscript to BioMed Central and we will help you at every step:}

- We accept pre-submission inquiries

- Our selector tool helps you to find the most relevant journal

- We provide round the clock customer support

- Convenient online submission

- Thorough peer review

- Inclusion in PubMed and all major indexing services

- Maximum visibility for your research

Submit your manuscript at www.biomedcentral.com/submit
Biomed Central 